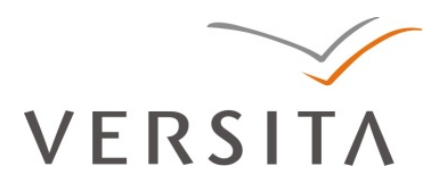

BULGARIAN ACADEMY OF SCIENCES

CYBERNETICS AND INFORMATION TECHNOLOGIES • Volume 13, No 4

Sofia $\bullet 2013$

Print ISSN: 1311-9702; Online ISSN: 1314-4081

DOI: $10.2478 /$ cait-2013-0053

\title{
Analytical Deriving of Second Order Model of Payne from First Order Lighthil-Whitham-Richards Model
}

\section{Vasilka Stoilova, Emil Nikolov, Nina Nikolova}

Industrial Automation, Faculty of Automatics, Technical University, 1000 Sofia

Emails:vassilka_stoilova@hotmail.comnicoloff@tu-sofia.bg ninan@tu-sofia.bg

Abstract: Analytical deriving has been realized to obtain the second order Payne model from the first order Lighthil-Witham-Richards model.

Keywords: First order model, second order model, traffic flow.

\section{First order Lighthill-Whitham-Richards model}

The first macroscopic models are given independently in the works of $\mathrm{L} \mathrm{ig} \mathrm{h} \mathrm{th} \mathrm{i} 11$ and $\mathrm{W} \mathrm{h} \mathrm{i} \mathrm{th} \mathrm{a} \mathrm{m} \mathrm{[20]} \mathrm{and} \mathrm{R} \mathrm{i} \mathrm{c} \mathrm{h} \mathrm{a} \mathrm{r} \mathrm{d} \mathrm{s} \mathrm{[26].} \mathrm{They} \mathrm{apply} \mathrm{the} \mathrm{analogy} \mathrm{between} \mathrm{the}$ flow of vehicles and the flow of a fluid to create a model with not so great complexity, but wide applicability. Lighthill-Whitham-Richards (LWR) model uses a fundamental relation (1), a conservation equation (2) and the assumption that the speed is a known density function (3). In fact (1) is an equation that is used to determine the vehicle flow, regardless of the road section considered and it includes the relationship between the flow rate and the density as function of the independent arguments - time and space.

$$
\begin{gathered}
q(x, t)=\rho(x, t) \cdot v(x, t), \\
\frac{\partial q(x, t)}{\partial x}+\frac{\partial \rho(x, t)}{\partial t}=0, \\
(x, t)=F(\rho(x, t)) .
\end{gathered}
$$

These models have many shortcomings $[6,24]$, which are based on many unrealistic assumptions. The first one is that speed adapts instantly to the desired speed and the flow at the exit of shock waves of congestion or the narrowing of the highway is equal to the infrastructure capacity. Another assumption is that the density of a shockwave of congestion has lower speed than the speed, where the 
density is lower. This means that the line will reach the body of the shock, which is unrealistic. LWR model is not able to cope with volatility, the type of traffic "stop and go" (sequences of acceleration and braking distances on congestions).

\subsection{First order model for the case with incoming and outgoing vehicles}

From the first order model LWR the objective relation (2) is given. It is for the case in which the traffic is one-way, with no entering or leaving vehicle on the highway. For the case in which there are incoming and outgoing vehicles from the highway the following dependence applies:

$$
\frac{\partial q(\rho(x, t))}{\partial x}+\frac{\partial \rho(x, t)}{\partial t}=r_{i}(x, t)-s_{i}(x, t)
$$

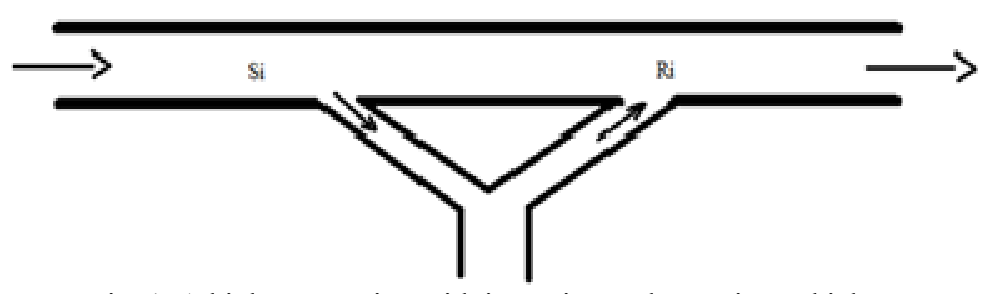

Fig. 1. A highway section with incoming and outgoing vehicles

where: $r_{i}(x, t)$ are outgoing vehicles, $s_{i}(x, t)$ are incoming vehicles, $N$ is number of vehicles, $d n / d t=q$ is flow rate, $d n / d x=\rho$ is density.

\subsection{Fundamental diagram of the traffic flow}

For each element of the infrastructure the parameters mean speed $\mathrm{v}$, density $\rho$ and flow rate $q$, which are the three most important variables, are related in a fundamental diagram, given in Fig. 2, which is analytically determined by the equation

$$
q(x, t)=\rho(x, t) \cdot F(\rho(x, t)) .
$$

The parameters in the description of the fundamental diagram are: the maximum density $\rho_{\max }$, free speed $v_{\mathrm{f}}$, maximum flow rate $q_{\max }$ and critical density $\rho_{\mathrm{cr}}$. The traffic flow maximum is observed as an optimal combination of the values of the other two parameters. The fundamental diagram depends on three parameters and must be presented in a three-dimensional space. However, for convenience, the following are used in the projection-plane: flow rate-density, density-speed and flow rate-speed. Normally flow-density is primarily used. The flow rate is determined by the value of the density and is used as an evaluation of the quality of the traffic flow for a segment of the highway (Fig. 2). There are two regions in the fundamental diagram: the traffic as a flow and congested traffic. The traffic flow corresponding to the running fluid is characterized by the velocity and movement of the vehicles, while in the congestion mode formations in lines with slow motion and consistent accelerations and stops are observed. Increasing the flow rate is suspended for the critical value of the density, which is determined by the capacity of the infrastructure, this is the maximum possible flow rate. For larger values of the density, the flow rate decreases until reset at the maximum value of the density. First $\mathrm{Gr}$ e e $\mathrm{n} \mathrm{s}$ h i e ld [11] has proposed a mathematical model of the fundamental 
diagram. He considers that the density and the flow rate linearly decrease, which leads to the parabolic shape of the fundamental diagram. Other forms of the fundamental diagram have been offered: a parabolic diagram - Greenshield, Masson; bi-parabolic diagram with horizontal tangent - Greenberg [10]; biparabolic diagram - B u is s on [2]; triangle diagram - C a s sidy [3]; trapezoidal diagram - D a g a n zo [4]; exponential diagram - Drake [7], P a p a g e o r g i o u [23]; curvilinear diagram - Cramer, Pipes. Various mathematical models have been proposed by various authors to describe the fundamental diagram [9]. They are formulated by using the free speed $v_{\mathrm{f}}$, maximum density $\rho_{\text {max }}$, critical density $\rho_{\text {cr }}$ and maximum flow rate $q_{\max }$. The most popular mathematical relationships are proposed by Greenshield [11], Greenberg [10]. Other models are made up by two separate parts for the two modes of traffic by May, Keller, and Van Aerde. The relationship between the speed $v$ and the density $\rho$ has certain formulations according to Chandler (see [8]), Drew (see [9]), Edie (see [10]) and Williams (see [8]), Drake (7).

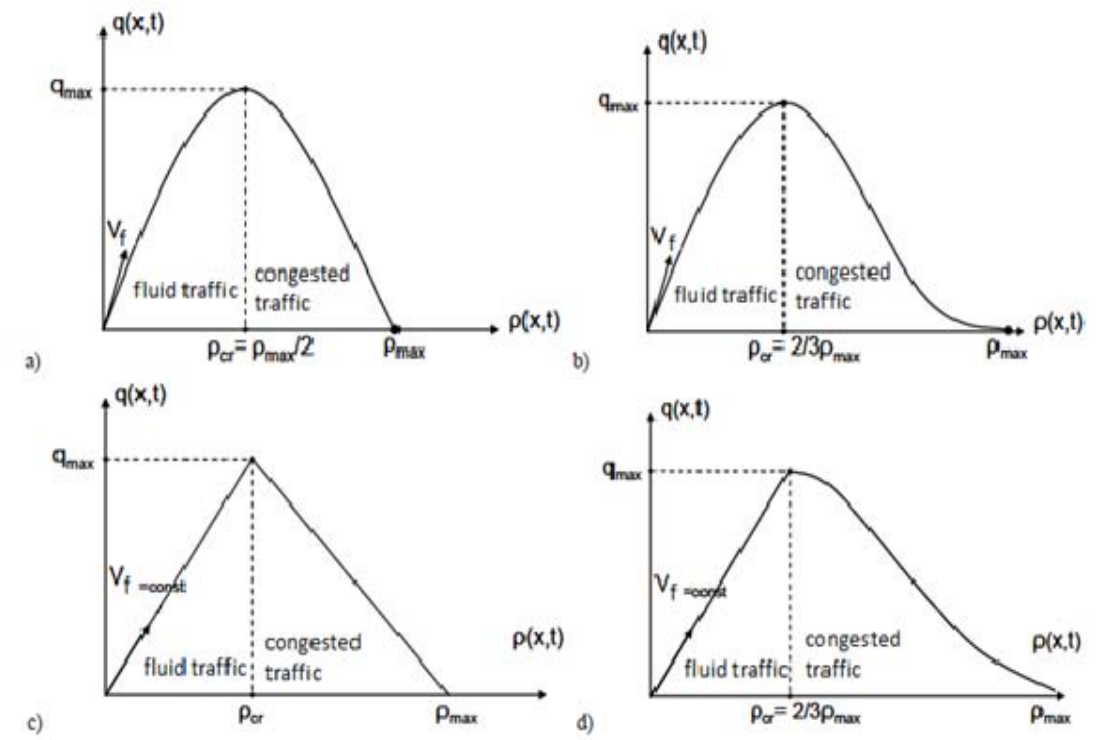

Fig. 2. Fundamental diagram according to: a) Greenshield; b) Drake; c) Cassidy; d) Greenberg

$$
\begin{gathered}
v(x, t)=v_{f}\left(1-\frac{\rho(x, t)}{\rho_{\max }}\right)-\text { Greenshield, } \\
v(x, t)=v_{f}\left(\ln \left(\frac{\rho(x, t)}{\rho_{\max }}\right)\right)-\text { Greenberg, } \\
v(x, t)=\frac{q_{\max }}{\rho(x, t)}\left(1-\frac{\rho(x, t)}{\rho_{\max }}\right)-\text { Chandler } \\
v(x, t)=v_{f}\left(1-\left(\frac{\rho(x, t)}{\rho_{\max }}\right)^{1 / 2}\right)-\text { Drew } \\
v(x, t)=v_{f} \cdot e^{\left(-\rho(x, t) / \rho_{\max }\right)}-\text { Edie } \\
v(x, t)=v_{f} \exp \left(\frac{1}{\alpha}-\left(\frac{\rho(x, t)}{\rho_{\alpha}}\right)^{\alpha}\right)-\text { Drake. }
\end{gathered}
$$


It is important to note that there is no universal fundamental diagram for any infrastructure. Any dependence above given is displayed for specific conditions and applied to specific cases.

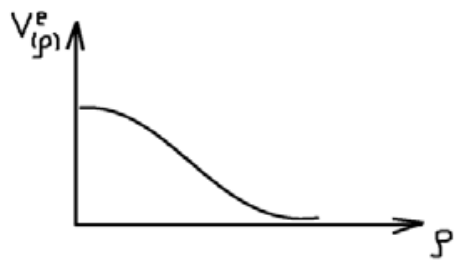

Fig. 3. Fundamental diagram density-speed

\section{Second order Payne type model}

The second order Payne-type models [23, 25] eliminate the previous three disadvantages by describing the speed variation in dynamics (12) accounting three additional processes. The first process is relaxation, which reflects the tendency of the driver to increase or decrease the velocity in order to reach your desired speed. The second component is convection, which represents the dependence of velocity on the moving vehicles entering and leaving a given segment from the highway. The third component is anticipation which consists of the change of the speed according to the conditions ahead. Essentially Payne uses a car-following model to develop his second order macroscopic model, according to which the drivers adapt their speed to a certain balance, which is a compromise between the desired and the actual speed according to the conditions in the area is

$$
\begin{gathered}
\frac{\partial v(x, t)}{\partial t}+\underbrace{v(x, t) \cdot \frac{\partial v(x, t)}{\partial x}}_{\text {convection }}=\underbrace{\frac{v_{e}(x, t)-v(\rho(x, t))}{T}}_{\text {relaxation }}-\underbrace{\frac{c_{o}^{2}(x, t)}{\rho(x, t)} \frac{\partial \rho(x, t)}{\partial x}}_{\text {anticipation }}, \\
c_{o}^{2}=-\frac{1}{T} \frac{d v_{e}(\rho(x, t))}{d \rho} .
\end{gathered}
$$

This model has also been criticized by Dagan zo [6], who noted that the component anticipation introduced in the model can lead to a negative flow rate when the derivatives in the space are big and it becomes unrealistic. Furthermore, he noted that the characteristics of the wave can become greater than the mean flow rate of the vehicles, which is not possible, since the distribution of the information is faster than the vehicles. One possible solution to this problem is given by P ap ag a orgiou [24], who proposes to accept a zero speed if the model gives negative values for the speed. He shows that the model uses the average speed. If the speed exceeds these average values it is good because the speed is real, but in a negative direction it becomes zero. The models developed on the kinetics of gas [12], represent an extension of LWR model, into which a restrictive equation for the speed change is introduced. Besides the conservation equation (1) [27] and the dynamic equation for the speed (12), these models apply an equation of restriction (dynamic equation for the speed)

$$
\frac{\partial \Theta}{\partial t}+v(x, t) \frac{\partial \Theta}{\partial x}=-2\left(\frac{P}{\rho}\right) \frac{\partial v}{\partial x}+2 \frac{\Theta_{e}-\Theta}{T}-\frac{1}{\rho} \frac{d J}{d x}, P=c_{o}^{2} \cdot \rho,
$$

where $P$ is the traffic pressure, $J$ is the covariance of the speed $\Theta$. 
In other models [13-17] in an invisible way microscopic parameters have been included, such as the length of the car, the reaction time of the driver, the dispersion speed, probability shifting on the lanes, acceleration time and others. These models take into account the same phenomenon in the space occupied by the car, so that it can never be empty. One example among hierarchical levels is a model given by (14) [29]:

$$
\begin{gathered}
v_{n}(t+T)=F\left(x_{n-1}(t)-x_{n}(t)\right)+G\left(x_{n-1}(t)-x_{n}(t)\right)= \\
=v_{e}(\rho(x, t)+\Delta x)+\alpha\left(\delta_{1} \frac{\partial v\left(x+\delta_{2}, t\right)}{\partial x}\right) ; 0<\delta_{2}<\delta_{1} .
\end{gathered}
$$

The function $F$ denotes the speed in balance, while $G$ reflects the linear reduction of the speed difference between the current car $n$ and the leading $n-1$. Another improvement to LWR model is made by D a g a n zo [5], Le sort [19] and Liu [21]. ARZ model developed by Aw-Rascle is seeking solutions of a partial differential equation of second order. A variable in the equation is the vehicle speed. Next equation is defined as a task of Rieman:

$$
\begin{aligned}
V_{t}+V V_{x}=\frac{V_{\mathrm{eq}}(\rho)-V}{\tau}+ & \rho P^{\prime}(\rho) \cdot V_{x} \Leftrightarrow \frac{\partial V}{\partial t}+\frac{\partial V}{\partial x}\left(V-\rho P^{\prime}(\rho)\right)= \\
& =\frac{1}{\tau}\left(V_{\mathrm{eq}}-V(\rho)\right),
\end{aligned}
$$

where $p^{\prime}(\rho)$ accounts the drivers' behavior.

The model of Zhang- Riemann task is solved by neglecting the condition for relaxation. The equation for speed variation becomes (16)

$$
\frac{\partial V}{\partial t}+\frac{\partial V}{\partial x}\left(V-\rho V_{\mathrm{e}}^{\prime}(\rho)\right)=0 .
$$

The model of Papageorgiou [22] and Kotsialos [18] is known as METANET. The relation is presented in a discrete form:

$$
\begin{gathered}
v_{m, i}(k+1)=v_{m, i}(k)+\frac{\Delta T_{\text {sim }}}{\tau_{m}}\left[V_{\mathrm{eq}}\left(\rho_{m, i}(k)\right)-v_{m, i}(k)\right]+ \\
+\frac{\Delta T_{\text {sim }}}{l_{m, i}} v_{m, i}(k)\left[v_{m, i-1}(k)-v_{m, i}(k)\right]-\frac{v_{m} \Delta T_{\text {sim }}\left[\rho_{m, i-1}(k)-\rho_{m, i}(k)\right]}{\tau_{m} l_{m, i}\left[\rho_{m, i}(k)-k_{m}\right]},
\end{gathered}
$$

where $\rho_{m, i}(k)$ is the density, $V_{m, i}(k)$ is the vehicle flow speed, $q_{m, i}(k)$ is the flow rate. Analytical models that take into account the change of lane geometry, add a component for the speed change in Payne model, presented in a discrete form. The second order models are made to predict the traffic when it is in balance. To take into account the conditions of imbalance, these models are complicated by additional components. The analytical solution of these models is complex, and therefore they are not always applied. The research directions are in the aspect of reporting and resolving the cases of congestion; cases to reduce the mean speed; to reduce the average time in the section of the highway [1], by setting parameters of the model. Restrictions requiring the traffic to behave like fluid are added. For practical considerations the following additional restrictions are imposed:

- The traffic flow on the lane must be below its maximum value. This condition is presented as follows:

$$
\begin{gathered}
\rho(x, t) \leq \rho_{\mathrm{cr}} \\
\rho(x, t) \leq 0.5 \rho_{\text {max }}-\text { according to Greenshield; }
\end{gathered}
$$




$$
\begin{gathered}
V(x, t) \geq V_{f}\left(1-\frac{0.5 \rho_{\max }}{\rho_{\max }}\right) \geq 0.5 V_{f} \\
V(x, t)=\left[V_{f} \div 0.5 V_{f}\right] \Longrightarrow \text { for } V_{\mathrm{cr}}=0.5 V_{f} \Rightarrow \rho(x, t)=\rho_{\mathrm{cr}} .
\end{gathered}
$$

- The maximum density that limits the maximum speed

$$
V_{f}(x, t)=V_{f} \rho(x, t) \Longrightarrow
$$

$$
\Rightarrow \rho(x, t) \rightarrow 1,\left(1-\frac{\rho(t)}{\rho_{\max }}\right) \rightarrow 1 \Rightarrow \frac{\rho(t)}{\rho_{\max }} \rightarrow 0 \Rightarrow \rho(t) \rightarrow 0 \Rightarrow V \rightarrow V_{f} \text {. }
$$

The traffic flow models of higher order in [28, 14] include specific phenomena, such as traffic "stop and go" compression and the narrowing of the highway, but these models are more mathematical, they are very complex and difficult to be used.

2.1. Demonstration of the second order Payne-type macroscopic level

Payne assumed a subjective dependence

$$
v(x,(t+T), t+T)=V^{e}(\rho(x+D))
$$

where $V^{e}$ is the static speed from the fundamental diagram, $D$ is the growth increment in the distance, $x(t)$ is the vehicle's location in time $t, v(x, t)$ is the actual speed, variable, $t+T$ is the reaction time.

$$
D=\frac{1}{\rho}
$$

Equation (20) defines that the future actual speed of the vehicle will respond to the static speeds, which define the fundamental diagram $V^{e}$.

Processing dependence (20):

- Decomposition in the order of Taylor's left part of (20) at point $(x, t)$. Decomposition in the growth increment $T$ :

$$
v(x,(t+T), t+T) \approx v(x, t)+T \cdot v(x, t) \cdot \frac{\partial v}{\partial x}+\frac{T \cdot \partial v(x, t)}{\partial t} .
$$

- Decomposition in the order of Taylor's right part of equation (20). Decomposition in the growth increment $D$ is

$$
V^{\mathrm{e}}(\rho(x+D))=V^{\mathrm{e}}(\rho(x, t))+D \cdot \frac{\partial \rho}{\partial x} \cdot \frac{\partial V^{\mathrm{e}}(\rho(x, t))}{\partial \rho} .
$$

From (22) and (23) (24) follows:

(24) $v(x, t)+T \cdot v(x, t) \cdot \frac{\partial v}{\partial x}+\frac{T \cdot \partial v(x, t)}{\partial t}=V^{e}(\rho(x, t))+D \cdot \frac{\partial \rho}{\partial x} \cdot \frac{\partial V^{e}(\rho(x, t))}{\partial \rho}$.

After taking into account the dependences (21) and (24), (25) follows:

(25) $v(x, t) \cdot \frac{\partial v(x, t)}{\partial x}+\frac{\partial v(x, t)}{\partial t}=\frac{V^{\mathrm{e}}(\rho(x, t))-v(x, t)}{T}+\frac{1}{\rho} \cdot \frac{\partial \rho(x, t)}{\partial x} \cdot \underbrace{\frac{\partial V^{\mathrm{e}}(\rho(x, t))}{\partial \rho}}_{C_{0}^{2}} \cdot \frac{1}{T}$.

After transformation of (25) and reporting the negative sign of the decreasing fundamental diagram (Fig. 3), (26) follows:

$$
\frac{\partial v(x, t)}{\partial t}+v(x, t) \cdot \frac{\partial v(x, t)}{\partial x}=\frac{V^{e}(\rho(x, t))-v(x, t)}{T}-\frac{C_{0}^{2}}{\rho} \cdot \frac{\partial \rho(x, t)}{\partial x} .
$$

That equation is equivalent to (12), which proves the second order Payne model. 


\section{Simulation}

A simulation has been made, which shows the dynamic step-response and impulse characteristics of a distributed parameter system (Fig. 4). The data used for the simulation are shown. Fig. 5 illustrates the dynamic step-response and impulse characteristics of the distributed parameter system with different length of the highway sections of one and $6 \mathrm{~km}$ with constant $\rho=0.4956$ and changing speed $V$.

$$
\stackrel{\rho_{\text {input }}(x, p)}{\longrightarrow} G(x, \xi, p) \stackrel{\rho_{\text {output }}(x, p)}{\longrightarrow}
$$

Fig. 4. Distributed parameter system

$$
\begin{gathered}
0 \mathrm{~km} / \mathrm{h} \leq V_{f} \leq 220 \mathrm{~km} / \mathrm{h}, \\
\rho_{j}=\rho_{\max }=160 \mathrm{veh} / \mathrm{km}, \\
0.15 \leq \rho^{0} \leq 0.4956 \\
\rho_{\text {cr }}=0.5 \rho_{\max }=80 \mathrm{veh} / \mathrm{km} ; \\
\rho_{\text {cr_ampe }}=0.1 \rho_{\max }=16 \mathrm{veh} / \mathrm{km}, \\
q_{\text {max }}=8800 \mathrm{veh} / \mathrm{h}, \\
0 \mathrm{~km} \quad \leq \leq 12 \mathrm{~km} .
\end{gathered}
$$
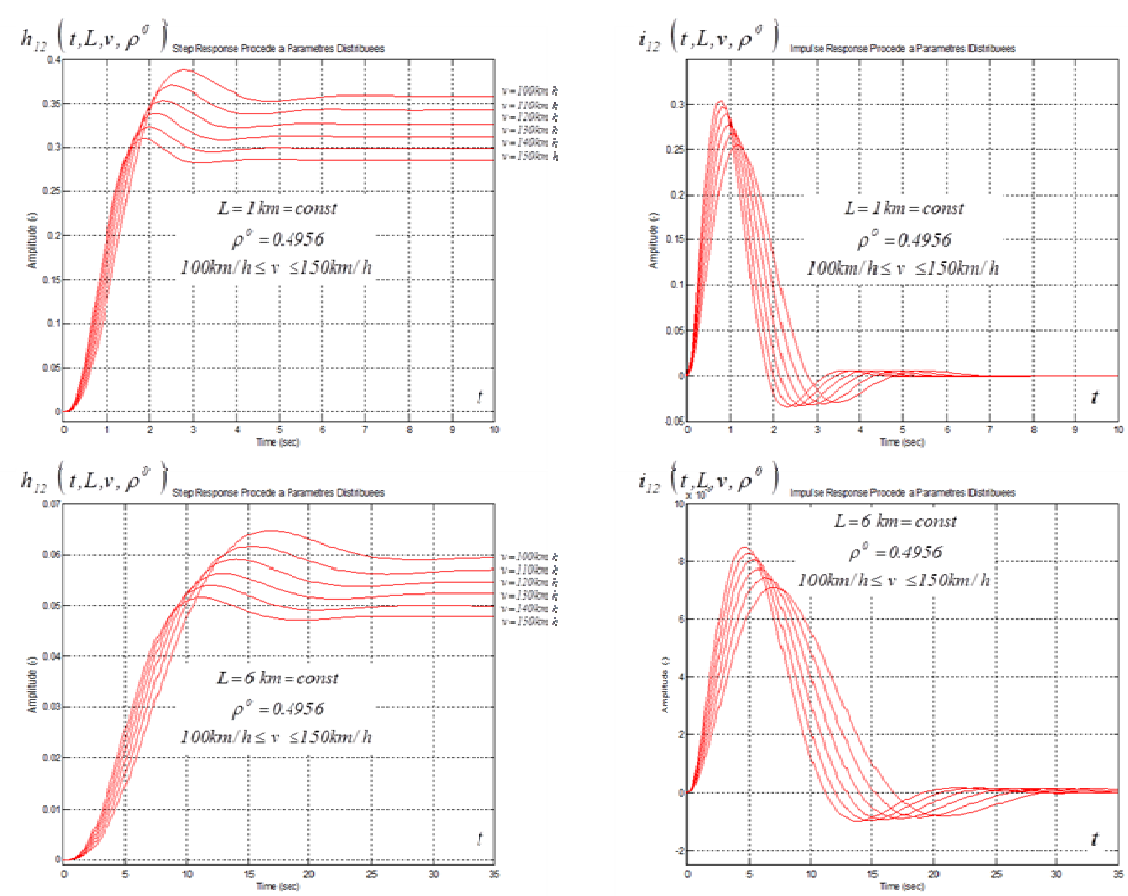

Fig. 5. Dynamic characteristics of the distributed parameter system 


\section{Conclusions}

The paper analyzes the shortcomings of the first order model of traffic dynamics on a highway stretch. Analytically the second order model has been derived. Numerical simulations of the dynamical behavior of the second order model have been performed. The step responses for this second order model have been evaluated. These results can be used for identification of the transfer function of a highway stretch. Thus, the relations from automatic control theory can be applied in the defining of the control influence of such distributed transportation systems.

Acknowledgement: The paper is partly supported by FP7 project 316087 ACOMIN "Advance computing and innovation".

\section{References}

1. B e 11 e mans, T. Traffic Control on Motorways. Dissertation Thesis, Katholieke Universiteit Leuven, D/2003/7515/25, ISBN 90-5682-413-9, 2003.

2. B u i s s o n, C. Analyse bibliographique et première classification des algorithmes de simulation de lécoulement du trafic routier. - Recherches transports et sécurité, 1996, No 53, 35-48.

3. Cassidy, M. J. Bivariate Relations in Nearly Stationary Highway Traffic Flow. Transportation Research, Vol. 32B, 1998, No 1, 49-59.

4. D a g a $\mathrm{n} \mathrm{z} \mathrm{o,} \mathrm{C.} \mathrm{F.} \mathrm{The} \mathrm{Cell} \mathrm{Transmission} \mathrm{Model:} \mathrm{A} \mathrm{Dynamic} \mathrm{Representation} \mathrm{of} \mathrm{Highway} \mathrm{Traffic}$ Consistent with the Hydrodynamic Theory. - Transportation Research, Part B, Vol. 28B, 1994, No 4, 269-287.

5. D a ga n z o, C. F. A Finite Difference Approximation of the Kinematic Wave Model of Traffic Flow. - Transportation Research, Part B, Vol. 29B,1995, No 4, 261-276.

6. Daganzo, C. F. Requiem for Second-Order Fluid Approximations of Traffic Flow. Transportation Research, Part B, Vol. 29B, 1995, No 4, 277-286.

7. D r a k e, J. A Statistical Analyses of Speed Density Hypotheses. Drake, J. S., J. L. Schofer, and A. D. May (1967). - Highway Research Record, Vol. 154, 1967, 53-87.

8. G a r t n e r, N. H. Development of Demand-Responsive Strategies for Urban Traffic Control. - In: Proceedings of the 1lthlFIP Conf. Syst. Modelling and Optimiz. P. Thoft-Christensen, Ed., New York, Springer-Verlag, 1984, 166-174.

9. G a r t n e r, N. H., C. J. M e s s e r, A. R a th i. Traffic Flow Theory: A State of the Art Report. US Project of: Department of Transportation Federal Highway Administration, Transportation Research Board, Oak Ridge National Laboratory, 1998. www.tfhrc.gov/its/tft/tft.htm trafficbook

10. Gre e $\mathrm{n}$ b e r g, H. An Analysis of Traffic Flow. - Operations Research, Vol. 7, January-February 1959, No 1, 79-85.

11. Greenshields, B. D. A Study of Traffic Capacity. - In: Highway Research Board Proceedings, Vol. 14, 1935, 448-477.

12. He l bing, D. Gas-Kinetic Derivation of Navier-Stokes-Like Traffic Equations. - Physical Review E, Vol. 53, 1996, No 3, 2266-2381.

13. H e 1 b i n g, D. Derivation, Properties, and Simulation of a Gas-Kinetic-Based, Nonlocal Traffic Model. - Physical Review E, Vol. 59, 1999, No 1, 239-253.

14. He l b ing, D., A. H e n n e k e, M. Tre iber. Phase Diagram of Traffic States in the Presence of Inhomogeneities. - Physical Review Letters, Vol. 82, May 1999, No 21, 4360-4363.

15. H e l b in g, D., M. S c h r e c k e n b e r g. Cellular Automata Simulating Experimental Properties of Traffic Flow. arXiv:cond-mat9812300v2, 21 Marth 1999.

16. Ho o g e nd o orn, S. P., P. H. L. B ovy. Modelling Multiple User-Class Traffic Flow. Transportation Research B, Vol. 34, 2000, No 2, 123-146. 
17. K 1 a r, A., W e g e n e r. A Hierarchy of Models for Multilane Vehicular Traffic I \& II: Modelling. - SIAM Journal of Applied Mathematics, 1998.

18. Kots ialos, A., M. Papage orgi ou, C. Diakaki, Y. Pavlis, F. Middelham. Traffic Flow Modelling of Large-Scale Motorway Networks Using the Macroscopic Modelling Tool METANET. - In: Recent Advances in Traffic Flow Modelling and Control, Proceedings of the Expert Seminar on Recent Advances in Traffic Flow. 1999.

19. Le s ort, J. B., J. P. L e b a c que. The Godunov Scheme and What is Means for First Order Traffic Flwo Models. - In: Proceedings of the 13th International Symposium on Transportation and Traffic Theory, Lyon, France, July 1996, 647-677.

20. L i g h th i 11, M. J., G. B. W h i th a m. On Kinematic Waves: I: Flow Movement in Long Rivers, II: A Theory of Traffic Flow on Long Crowded Roads. - In: Proceedings of the Royal Society: A229, London, 1955.

21. Liu, G., A. S. Lyrintzis, P. G. Michalopoulos. Improved High-Order Model for Freeway Traffic Flow. - Transportation Research Record, Vol. 1644, 1998, 37- 46.

22. P a p a g e o r g i o u, M. Application of Automatic Control Concept to Traffic Flow Modeling and Control. - In: A. V. Balakrishan, M. Thoma, Eds. Lecture Notes in Control and Information Sciences. Berlin, Springer, 1983, 4-41.

23. Papag e orgiou, M. Dynamic Modeling, Assignment, and Route Guidance in Traffic Networks. - Transportation Research, Part B, Vol. 24B, 1990, No 6, 471-495.

24. P a p a g e o r g i o u, M. Some Remarks on Macroscopic Traffic Flow Modelling. - Transportation Research, Part A, Vol. 32, 1998, No 5, 323-329.

25. P a y n e, H. J. Models of Freeway Traffic and Control. - In: Simulation Council Proceedings, 1971, No 1, 51-61.

26. R i c h a r d s, P. I. Shock Waves on the Highway. - Operations Research, Vol. 4, 1956, $42-57$.

27. S to il ova, V. Methods for Modelling Transport Traffic in Highways - Part I. - In: Proc. of International Conf. Automatics and Informatics, Sofia, 2013.

28. Treiber, M., A. Hennecke, D. Helbing. Congested Traffic States in Empirical Observations and Microscopic Simulations. - Physical Review E, Vol. 62, August 2000, No 2, 1805-1824.

29. Z h a n g, H. M. Structural Properties of Solutions Arising from a Nonequilibrium Traffic Flow Theory. - Transportation Research, Part B, Vol. 34, 2000, 583-603. 